\title{
A photo-responsive modulation of trans-zeatin
}

Xin Sun, ${ }^{\mathrm{a}, \mathrm{b}}$ Li Liu, ${ }^{\mathrm{a}, \mathrm{b}}$ and Liang Cheng, ${ }^{\mathrm{a}, \mathrm{b}, *}$

${ }^{a}$ Beijing National Laboratory for Molecular Sciences (BNLMS), CAS Key Laboratory of Molecular Recognition and Function, CAS Research/Education Center for Excellence in Molecular Sciences, Institute of Chemistry, Chinese Academy of Sciences, Beijing 100190, China.

${ }^{b}$ University of Chinese Academy of Sciences, Beijing 100049, China.

* cheng1@iccas.ac.cn

\section{ABSTRACT}

Photo-irradiation of an appropriately designed caged hormones enables the control and manipulation of the corresponding biological processes with high spatial and temporal resolution. Caged transzeatin of various types of nitrobenzene carbonate related photoremovable protecting groups have been synthesized. A rapid irradiation liberates the trapped trans-zeatin molecule, permitting targeted perturbation of biological processes including degradation, glucosylation and recognition by appropriate enzymes.

Keywords

Cytokinin; zeatin; decaging; photo-irradiation

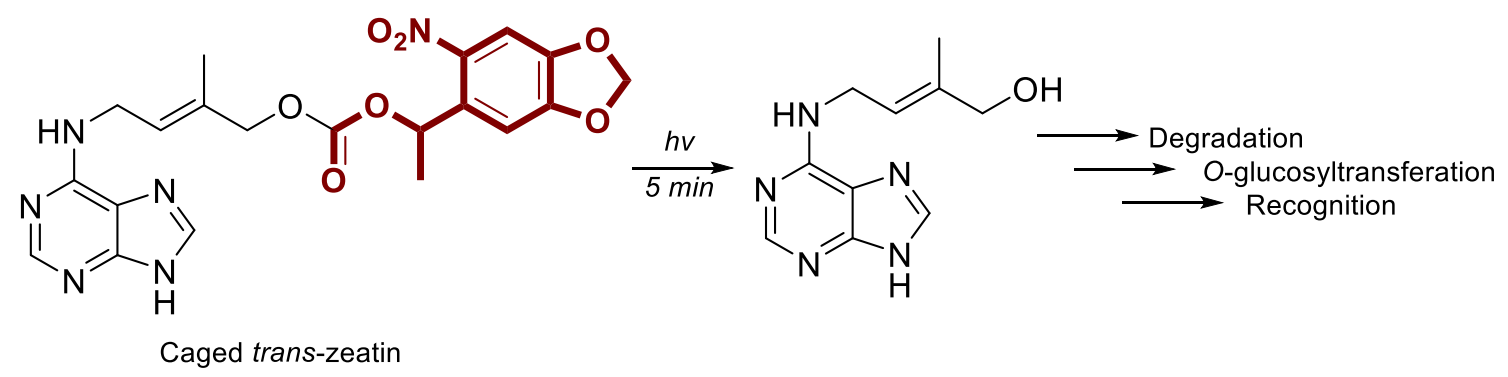


Without efficient communication among cell, tissue and organs, the growth and development of plants are impossible. This communication coordinates through chemical signals from one part of the plant to another. Hormones through interacting with receptor proteins in cells modulate cellular processes via transcriptional or post transcriptional events that ultimately induce physiological or developmental response. ${ }^{[1-2]}$ Among the growing list of plant hormones, cytokinins is a unique group of small organic molecules that is well known for their cell-division promoting capability of plant cells. ${ }^{[3]}$ The most common class of cytokinins has isoprenoid side chains, including isopentenyl adenine (iP), dehydrozeatin (DHZ), and trans-zeatin (tZ). Acquired information about their biological activities, interconversions, and metabolism has significantly improved understanding of their mechanisms of action and functions in planta. So far, cytokinins have been shown to have effects on many physiological and developmental processes, including leaf senescence, apical dominance, formation and activity of apical meristem and vascular development. The last three processes are critical during the course of plant cell, tissue and organ culture of plants. Although numerous genetic transformation methods have been developed which offer an important option for effective multiplication of cytokinins within a limited time frame, ${ }^{[4-7]}$ their use in cellular conditions or even on a whole plant scale is often limited by the compensation of multiple signalling pathways, i.e., the biosynthesis and inactivation of cytokinins with a subfamily of enzymes. To circumvent this problem, different methods are highly required that would allow for a more precise manipulation of the cytokinin levels. ${ }^{[8-9]}$


with its $c$ is-isomer $(\mathrm{cZ})^{[10]}$ and conjugates. It is thought to play a central physiological role as well as one of the major hormones, which performs a vital role in plant growth and development, and 
regulates many important physiological processes, such as apical dominance, root development, reproduction and senescence. Currently, two different pathways have been identified for the biosynthesis of $\mathrm{tZ}$ : the tRNA pathway and the AMP pathway. In the former pathway $\mathrm{tZ}$ is a recycled product of isopentenylated tRNAs $\left(i^{6} \mathrm{~A}\right)$, while in the latter it is synthesized from an isopentenyl donor, dimethylallyl diphosphate (DMAPP), and AMP, ADP, or ATP by isopentenyltransferases. ${ }^{[11]}$ Cytokinin hydroxylases promote the final hydroxylation step on isopentenylated adenosines, converting them to $\mathrm{tZ}$ or its analogues. ${ }^{[12]}$ Although the enzymatic regulation of $\mathrm{tZ}$ metabolism has made some progress in the past, ${ }^{[13-16]}$ a spatiotemporal manipulation of $\mathrm{tZ}$ without interfering with the genetic redundancy would be of benefit for providing new insights into the metabolism and role of $\mathrm{tZ}$.

In our continuance of exploring photo-chemical tools for the precise modulation of ribonucleic acid epigenetics, ${ }^{[17-19]}$ we envisioned that a sophisticatedly modified zeatin derivative may temporarily cover its activity from interacting functionally with proteins, either acting as ligands for binding receptors, inhibiting degradation enzymes or blocking metabolism protein interactions, etc. However, its biophysical properties may be switched by external stimuli by releasing the active hormone, which binds to the signalling proteins and subsequently transmit the signal through a sequence. ${ }^{[9,20-21]}$ Considering the fact that $\mathrm{tZ}$ is characterized by the hydroxyl group at the end of the prenyl side chain and the stereo-isomeric position of this hydroxyl group, we expected that a probably selected photo-caging group at the hydroxyl ${ }^{[22]}$ may result in an inactive form and then be activated by light irradiation when requested (Figure 1A). ${ }^{[23]}$ The activity-turning on in space-time would be especially attractive for a precise delineation of its function in biological processes. 
(A)

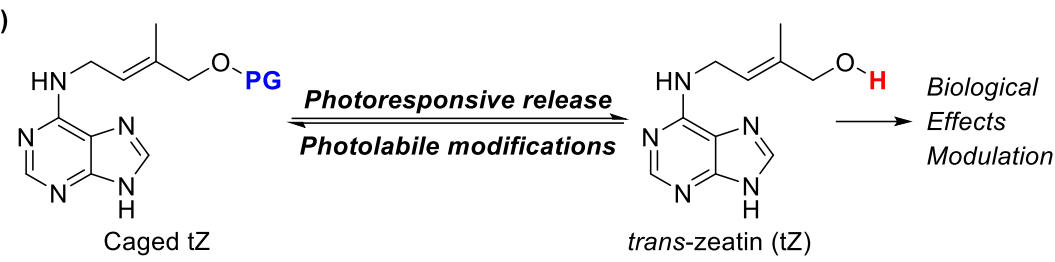

(B)<smiles>C/C(=C\CNc1ncnc2[nH]cnc12)COC(=O)OC(C)c1cc2c(cc1[N+](=O)[O-])OCO2</smiles><smiles>C/C(=C\CNc1ncnc2[nH]cnc12)COC(=O)OCc1cc2c(cc1[N+](=O)[O-])OCO2</smiles><smiles>C/C(=C\CNc1ncnc2[nH]cnc12)COC(=O)OC(C)c1ccccc1[N+](=O)[O-]</smiles><smiles>C/C(=C\CNc1ncnc2[nH]cnc12)COC(=O)OCc1ccccc1[N+](=O)[O-]</smiles>

Figure 1. (A) Schematic demonstration of $\mathrm{tZ}$ modification and its photo-modulation. (B) Chemical structures of synthetic photo-caged $\mathrm{tZ}$ derivatives.

For this purpose, we designed and synthesized four $\mathrm{tZ}$ derivatives as shown in Figure $2 \mathrm{~B}$. The terminal hydroxyl group can be smoothly substituted with $o$-nitrophenyl benzyl carbonates derived from piperonyl alcohol or benzyl alcohol as photo-removable protecting groups ${ }^{[24]}$ (see SI for details). To our delight, all those caged $\mathrm{tZ}$ molecules were susceptible to an extremely rapid cleavage under the irradiation of ultra-violent light $\left(365 \mathrm{~nm}, 100 \mathrm{~mW} \cdot \mathrm{cm}^{-2}\right)$ in an aqueous solution within 4 minutes (Figure 2B). Compounds 1 and $\mathbf{3}$ were among the fastest photo-decaging group, in which 2 minutes of irradiation led to complete cleavage. A notable generation of $\mathrm{tZ}$ was observed from the high performance liquid chromatography (HPLC) analysis (Figure 2C). In all cases, a quantitative amount of $\mathrm{tZ}$ was detected in 3 minutes. Caged $\mathrm{tZ}$ molecule $\mathbf{1}$ was chosen as the lead compound as it afforded $\mathrm{tZ}$ in an over $90 \%$ yield upon photo-irradiation, which would benefit further application in live organisms as it may eliminate the light damage caused by longtime exposure to light. In order to exclude that the rapid release of $\mathrm{tZ}$ was caused by lightpromoted isomerization of nitrobenzene motif, the compound $\mathbf{1}$ was subjected to the hydrolysis in 
dark conditions (Figure 2D). Although the carbonate linkage always exhibited less stability toward alkaline hydrolysis, ${ }^{[25]}$ it is, however, relatively stable under the physiological conditions. No apparent hydrolysis was observed even after 30 days' incubation, indicating that the compound may stay inert without the external light trigger.

(A)

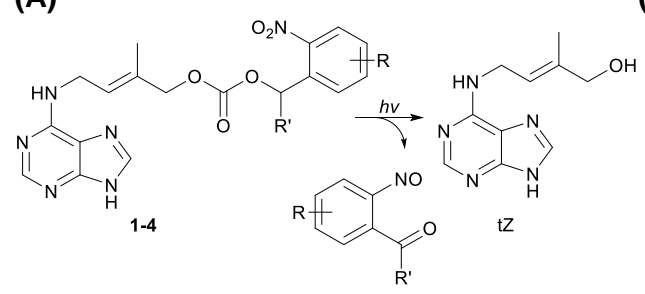

(C)

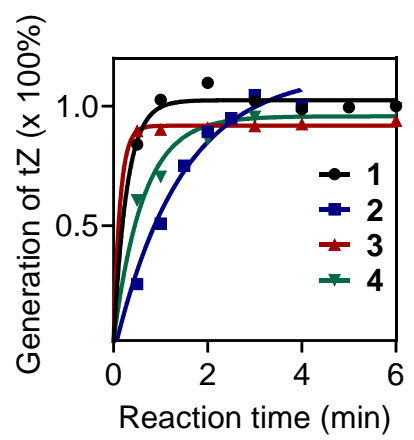

(B)

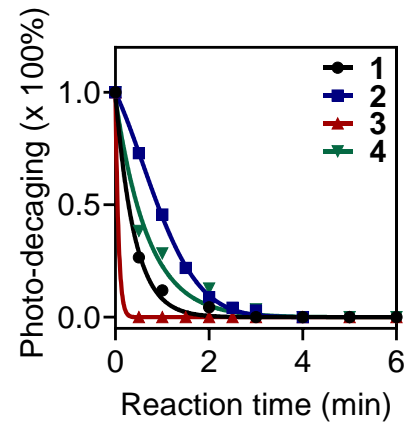

(D)

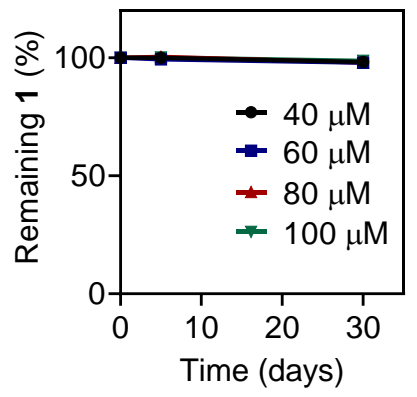

Figure 2. (A) Photo-decaging of $\mathrm{tZ}$ derivatives 1-4 for the release of $\mathrm{t} Z$ with the side products nitrosobenzenes. (B) Photo-decaging efficiency of 1-4 upon light irradiation. 1: Black line embed with circles; 2: Navy blue line embed with squares; 3: Red line embed with upward triangles; 4:

Green line embed with downward triangles. (C) Efficiency of $\mathrm{tZ}$ generation upon light irradiation of 1-4. (D) Stability of compound $\mathbf{1}$ in an aqueous solution and in dark conditions. The reaction was repeated under four concentrations ( $40 \mu \mathrm{M}, 60 \mu \mathrm{M}, 80 \mu \mathrm{M}$ and $100 \mu \mathrm{M}$, respectively).

With the promising molecule $\mathbf{1}$ in hand, we next investigated its activity-switch effect upon enzymatic degradation (Figure 3). Inactivating cytokinins by irreversibly cleaving their $N^{6}$ side chains to generate adenine or adenosine. ${ }^{[26-27]}$ The endogenous level of $\mathrm{tZ}$ in plant cells is regulated 
in part by degradation via $\mathrm{CKX}$ through the recognition of the unsaturated double bond within the prenyl substituent. We predicted that the large and hydrophobic $o$-nitrophenyl benzyl group located at the terminal prenyl group may interfere with the CKX enzyme, prohibiting the degradation and thus remain intact (Figure 3A). In this regard, we expressed and purified the rice (Oryza sativa L.) cytokinin oxidase/dehydrogenase OsCKX11. The degradation activity of the recombinant protein was not affected by ultra-violent light ( 5 minutes, $10 \mathrm{~mW})$. However, the compound 1 was considerably decaged in that time-frame, delivering $\mathrm{tZ}$ in a $66 \%$ yield. We were delighted to find that the carbonate $\mathbf{1}$ was not oxidized or hydrolyzed by OsCKX11. No tZ or adenine was detected from HPLC analysis, suggesting that the dehydrogenase is indeed sensitive to the subtle change at the prenyl substituent even with the presence of $\mathrm{C}=\mathrm{C}$ double bond (Figures $3 \mathrm{~B}$ C). ${ }^{[28]}$ Nonetheless, it rapidly recognized the released $t Z$ upon a short time of light exposure and smoothly converted it into the natural adenine. A quantitative amount of adenine was observed, indicating that the oxidative degradation was not effected by ultra-violent irradiation (Figure 3C). Thus we conclude that the degradation liability was totally blocked in compound $\mathbf{1}$ and can be efficiently turned-on by light. 
(A)
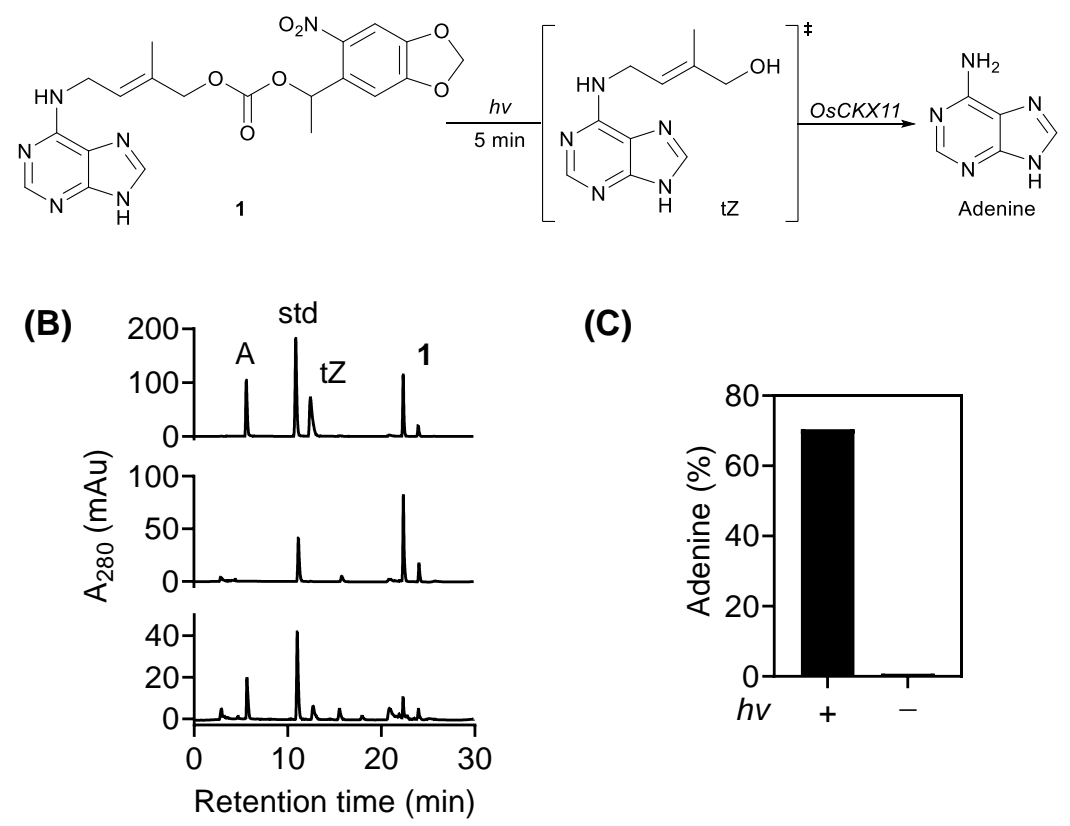

(C)

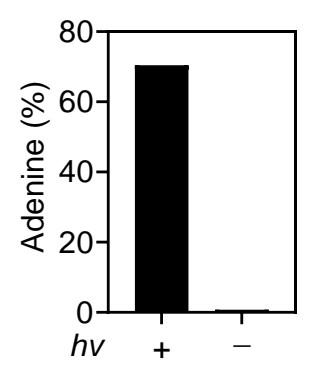

Figure 3. (A) Photo decaging of compound 1 and subsequent oxidative degradation to adenine upon incubation of OsCKX11. (B) HPLC traces for the detection of compound $\mathbf{1}$ before and after light irradiation. From up to down: the mixture of adenine (A), standard (std, $N^{6}$-methyl adenine), tZ and $\mathbf{1}$; incubation of compound $\mathbf{1}$ with OsCKX11 in dark conditions; and the reaction mixture after light irradiation of 5 minutes. (C) The calculation of adenine generation with and without light irradiation. All raw data were listed in SI.

$O$-glucosylation, in which a glucose molecule is attached to the hydroxyl group of isoprenoid $N^{6}$ side chain, is thought to be one of the main metabolism pathways for $\mathrm{t} Z$ and other zeatin-type cytokinins (DHZ, cZ, etc). ${ }^{[29]}$ The trans-zeatin $O$-glucosyltransferase of Arabidopsis thaliana, UGT85A1, was previously identified as one of the key enzymes for this process. ${ }^{[30]}$ The generated cytokinin $O$-glucosides, i.e., tZOG, was not able to activate cytokinin receptors AHK3 and AHK4. ${ }^{[31-32]}$ Nevertheless, the covalent $O$-glucoside conjugate can be cleaved by endogenous hydrolase like $\beta$-glucosidase to release the biologically active hormone $\mathrm{tZ}$. We predicted that the absence of terminal hydroxyl in compound 1 would hinder further $O$-glucosylation with UGT85A1. 
Upon light irradiation, the $O$-glucosyltransferase will then activate the generated $\mathrm{tZ}$ intermediate and furnish the $O$-glucoside product tZOG (Figure 4A). Indeed, the recombinant protein UGT85A1 smoothly converted the free $\mathrm{tZ}$ to the dSIgnated conjugate tZOG, but showed no activity towards $\mathbf{1}$ (Figure 4B). Interestingly, when the same mixture was exposed to light, no tZOG was detected from HPLC analysis. However, only the decaged intermediate $\mathrm{tZ}$ was observed. In order to identify the reasons for the inaction of UGT85A1 under the circumstance, a mixture with free $\mathrm{tZ}$ was subjected to the same condition. The title product $\mathrm{ZZOG}$ was perceived as expected, indicating that the light exposure was not the cause of inaction of the UGT85A1. Surprisingly, when the starting material piperonyl alcohol 5 and the corresponding by-product $\mathbf{6}$ in the photo-cleavage of $\mathbf{1}$ (Figure 4C) was mixed with $\mathrm{tZ}$ and then incubated with UGT85A1 in dark conditions, a very different phenomenon was observed (Figure 4D). The $O$-glucosylation with alcohol 5 went well, affording the product $\mathrm{tZOG}$ in an inferior yield. However, when the nitrosobenzene by-product 6 was used, a dramatic decrease of tZOG was noticed, suggesting that the glucosyl-transferation ability of UGT85A1 was totally inhibited. We conducted a series of control experiments (Figure S4, SI). It turned out the nitrosobenzene motif, not the carbonyl functionality, was responsible for the activity depression of $O$-glucosyltransferase, probably due to the oxidative dimerization of cysteine residues by reacting with the reactive nitroso group. ${ }^{[33-34]}$ Although highly effective inhibitors of $N$ glucosyltransferases, an analogue of $O$-glucosyltransferase, were identified, those compounds tentatively dominated the binding sites within the glucosyl-transferation cavity in non-covalent interactions. ${ }^{[35]}$ Accordingly, the current study may have shield some insights on the discovery of covalent inhibitor for $O$-glucosyltransferases. 
(A)



(B)
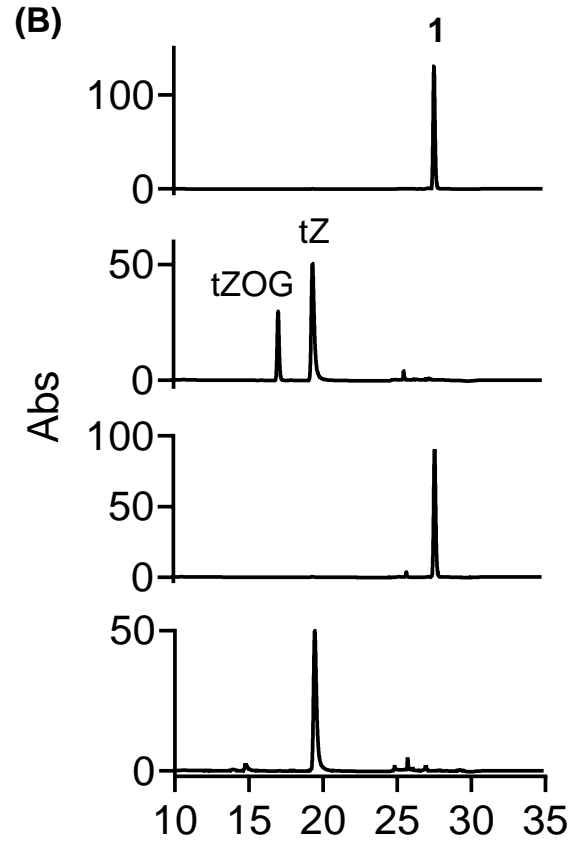

Retention time (min)
(C)
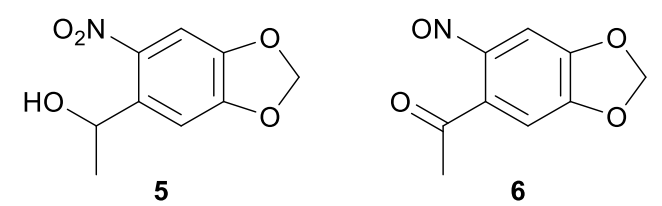

6

(D)

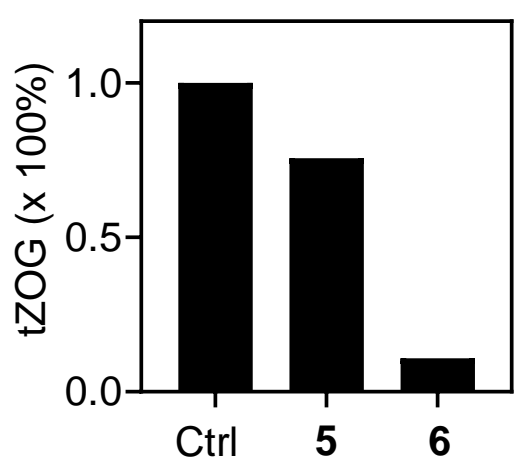

Figure 4. (A) Photo decaging of compound 1 and subsequent glycosylation to $\mathrm{Z}$ - $O$-glucoside (tZOG) upon incubation of UGT85A1. (B) HPLC traces for the detection of tZOG before and after light irradiation with compound 1. From up to down: the compound 1; a mixture of adenine $\mathrm{tZ}$ and tZOG; incubation of compound 1 with UGT85A1 in dark conditions; and the reaction mixture after light irradiation of 5 minutes. (C) The structure of the starting material piperonyl alcohol 5 and the corresponding by product 6 in the photo-cleavage of $1 . \quad$ (D) Calculation of $\mathrm{tZOG}$ generation of $\mathrm{tZ}$ under the promotion of UGT85A1 with the incubation of $\mathbf{5}$ or $\mathbf{6}$. All raw data were listed in SI.

There are three membrane-located cytokinin receptors (AHK2, AHK3 and CRE1/AHK4) in Arabidopsis thaliana that are sensor histidine kinases containing a ligand-binding CHASE domain. It was recently suggested that the AHK4 and its homologues are receptor kinases that can transduce 
tZ signal across the plasma membrane of $A$. thaliana. ${ }^{[36]}$ In order to verify the feasibility of the photo-caging strategy for temporarily blocking AHK4 recognition, we conducted a series of computational simulation (Figure 5). We used Autodock 4.2 to dock the parent molecule $\mathrm{tZ}$ with AHK4 (Figure 5A). The results were consistent with the previous reports that The $N^{7}$ atom on the $\mathrm{tZ}$ adenine ring formed a hydrogen bond with $\mathrm{Asp}^{262}$ residue, while the terminal hydroxyl group at the side chain formed another hydrogen bond with $\mathrm{Thr}^{294}$ residue. ${ }^{[37]}$ Those two key interactions facilitated the appropriate recognition of AHK4 with the $\mathrm{tZ}$ ligand. However, when the terminal hydroxyl was substituted with a large phenyl group, the hydrogen bond with $\mathrm{Thr}^{294}$ was abolished, with surrogate but weak interactions from the carbonate or nitro group to the residues within the cavity (Figure 4C). The inferior recognition of $\mathbf{1}$ was also established by the careful calculation of binding energies with AHK4 (Figure 4D). The net Gibbs free energy $(\Delta \mathrm{G})$, inhibitor constant $\left(K_{\mathrm{i}}\right)$, and ligand efficiency (LE) all indicated that compound $\mathbf{1}$ may not the ligand for activating AHK4, and thus can not trigger the signal transfer.

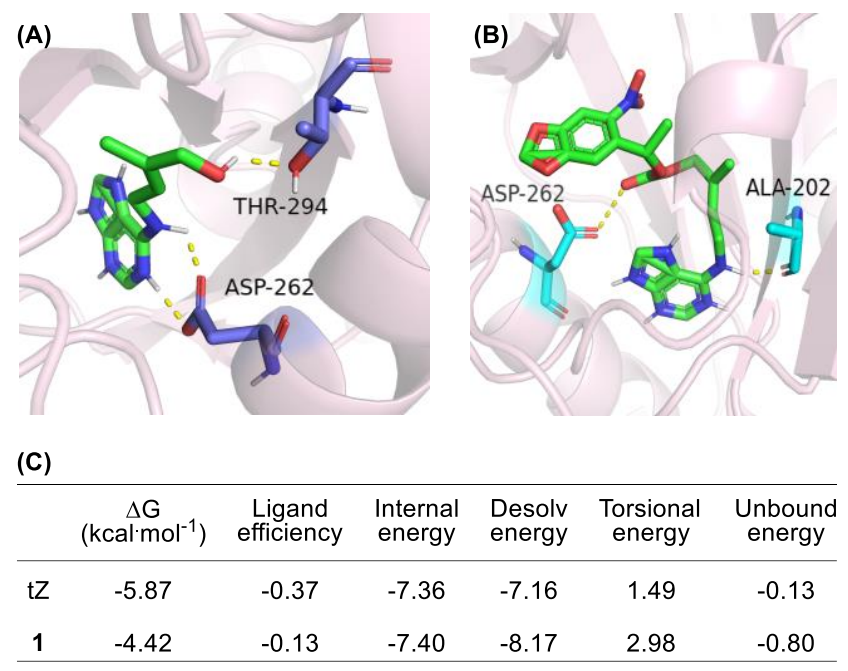

Figure 5. (A) The protein AHK4 (PDB: 3T4Q) docking prediction with tZ. Dash lines indicate possible interactions. (B) The protein AHK4 docking prediction with compound 1. (C) Energy calculation results for docking prediction with $\mathrm{tZ}$ and 1, respectively. For docking procedures and detailed results, see SI. 
In conclusion, we have achieved the first light response modification of trans-zeatin. The synthesized caged derivatives exhibited considerable stability and yet remained high sensitivity towards ultra-violent light irradiation. The photo-modulation has enabled a rapid release of $\mathrm{tZ}$ under physiological conditions and activity turn-on towards dehydrogenase. A covalent inhibitory of nitrosobenzene with $O$-glucosyltransferase was also unexpectedly discovered. Finally, the molecular docking with the caged compound was performed to verify the interference of binding activity. To date, few examples of in vivo applications of photo-caging techniques have been reported for cytokinin studies. What is most challenging is a new caged compound that is capable of being used in whole plant or organisms without perturbing the other proteins. To attain this goal, a new stimuli-removable protecting group would be desirable.

\section{ACKNOWLEDGEMENTS}

We thank Prof. Ke-Wei Zhang (Zhejiang Normal University) for providing us with the CKX11 plasmid and Dr. Tian-Qi Zhang (Institute of Plant Physiology and Ecology (SIPPE), Chinese Academy of Sciences) for helpful discussions. This work was supported by the National Key R\&D Program of China (2017YFA0208100 and 2020YFA0707901), National Natural Science Foundation of China $(22022704,21977097)$ and Chinese Academy of Sciences.

\section{References}

[1] Anfang, and E. Shani, Curr. Opin. Plant Biol., 2021, 63, 102055.

[2] M.K. Jiang, and T. Asami, Biosci. Biotechnol. Biochem., 2018, 82, 1265.

[3] D. W. S. Mok, and M. C. Mok, Cytokinins: chemistry, activity, and function, 1994.

[4] Q. H. Ma, Crit. Rev. Biotechnol., 2008, 28, 213. 
[5] P. Hedden, and A. L. Phillips, Curr. Opin. Biotechnol., 2000, 11, 130.

[6] D. Zalabák, H. Pospíšilová, M. Šmehilová, K. Mrízová, I. Frébort, and P. Galuszka, Biotechnol. $A d v ., 2013, \mathbf{3 1}, 97$.

[7] S. Gajdošová, L. Spíchal, M. Kamínek, K. Hoyerová, O. Novák, P. I. Dobrev, P. Galuszka, P. Klíma, A. Gaudinová, and E. Žižková, J. Exp. Bot., 2011, 62, 2827.

[8] M. Schäfer, and S. Meldau, Methods Mol. Biol., 2017, 1569, 127.

[9] A. Rigal, Q. Ma, and S. Robert, Front. Plant Sci., 2014, 5, 373.

[10] M. Schäfer, C. Brütting, I. D. Meza-Canales, D. K. Großkinsky, R. Vankova, I. T. Baldwin, and S. Meldau, J. Exp. Bot., 2015, 66, 4873.

[11] K. Tatsuo, Plant Cell Physiol., 2001, 7, 677.

[12] K. Takei, T. Yamaya, and H. Sakakibara, J. Biol. Chem., 2004, 279, 41866.

[13] B. Wolfram, R. Eswarayya, and H. Alexander, S. Thomas, Front. Plant Sci., 2012, 3, 8.

[14] T. Raines, Plant J., 2016, 85, 134.

[15] A. P. Rodó, N. Brugière, R. Vankova, J. Malbeck, J. M. Olson, S. C. Haines, R. C. Martin, J. E. Habben, D. W. S. Mok, and M. C. Mok, J. Exp. Bot., 2008, 59, 2673.

[16] C. Li, Z. Lu, Q. Fu, and F. -X. Zeng, PeerJ, 2018, 6, e4812.

[17] L.-J. Xie, X.-T. Yang, R.-L. Wang, H.-P. Cheng, Z.-Y. Li, L. Liu, L.-Q. Mao, M. Wang, and L. Cheng, Angew. Chem. Int. Ed., 2019, 58, 5028.

[18] L. Lan, Y.-J. Sun, X.-Y. Jin, L.-J. Xie, L. Liu, and L. Cheng, Angew. Chem. Int. Ed., 2021, 60, 18116.

[19] H.-P. Cheng, X.-H. Yang, L. Lan, L.-J Xie, C. Chen, C.-M. Liu, J.-F. Chu, Z.-Y. Li, L. Liu, T.-Q. Zhang, D.-Q. Luo, and L. Cheng, Angew. Chem. Int. Ed., 2020, 59, 10645.

[20] G. C. and Ellis-Davies, Nat. Methods, 2007, 4, 619.

[21] N. Hemelíková, A. Žukauskaitė, T. Pospíšil, M. Strnad, K. Doležal, and V. Mik, J. Agric. 
Food Chem., 2021, 69, 12111.

[22] Y.-J. Sun, L. Liu, and L. Cheng. Chem. Comm., 2020, 56, 6484.

[23] K. I. Hayashi, N. Kusaka, K. Ando, T. Mitsui, T. Aoyama, and H. Nozaki, Bioorg. Med. Chem. Lett., 2012, 22, 5663.

[24] S. G. Richard, R. S. Givens, P. G. Conrad, Abraham L. Yousef, and J. Lee, CRC Handbook of Organic Photochemistry and Photobiolog, 2004.

[25] L.W. Dittert, and T. Higuchi, J. Pharm. Sci., 2010, 52, 852

[26] W. Zhang, K. Peng, F. Cui, D.-L. Wang, J.-Z. Zhao, Y.-J. Zhang, N.-N. Yu, Y.-Y. Wang, D.-

L. Zeng, Y.-H. Wang, Z.-K. Cheng, and K.-W. Zhang, Plant Biotechnol. J., 2020, 19, 335.

[27] P. Galuszka, I. Frébort, M. Šebela and P. Peč, Plant Growth. Regul., 2000, 32, 315.

[28] T. Werner, I. Köllmer, I. Bartrina, K. Holst, and T. Schmülling, Plant Biol., 2006, 8, 371.

[29] D. W. Mok, and M. C. Mok, Annu. Rev. Plant Physiol. Plant Mol. Biol., 2001, 52, 89.

[30] H.-J. Shang, X.-M Ma, M. Kojima, H. Sakakibara, Y.-W Wang and B.-K. Hou, Planta, 2013, $237,991$.

[31] L. Spíchal, N. Yu. Rakova, M. Riefler, T. Mizuno, G. A. Romanov, M. Strnad, and T. Schmülling, Plant Cell Physiol., 2004, 45, 1299.

[32] G. A. Romanov, S. N. Lomin, and S. Thomas, J. Exp. Bot., 2006, 57, 4051.

[33] S. Kazanis, and R. A. Mcclelland, J. Am. Chem. Soc., 1992, 114, 3052.

[34] A. Barth, J. Corrie, and M. J. Gradwell, J. Am. Chem. Soc., 1997, 119, 4149.

[35] B. Hou, E. K. Lim, G. S. Higgins, and D. J. Bowles, J. Biol. Chem., 2004, 279, 47822.

[36] H. Yamada, T. Suzuki, K. Terada, K. Takei, K. Ishikawa, K. Miwa, T. Yamashino, and T.

Mizuno, Plant Cell Physiol., 2001, 42, 1017.

[37] M. Hothorn, T. Dabi, and J. Chory, Nat. Chem. Biol., 2011, 7, 766. 